
LA-8442-MS

Informal Report

UC-28

Issued: July 1980

\title{
Matching Bunched Beams to Alternating Gradient Focusing Systems
}

\author{
W. P. Lysenko
}
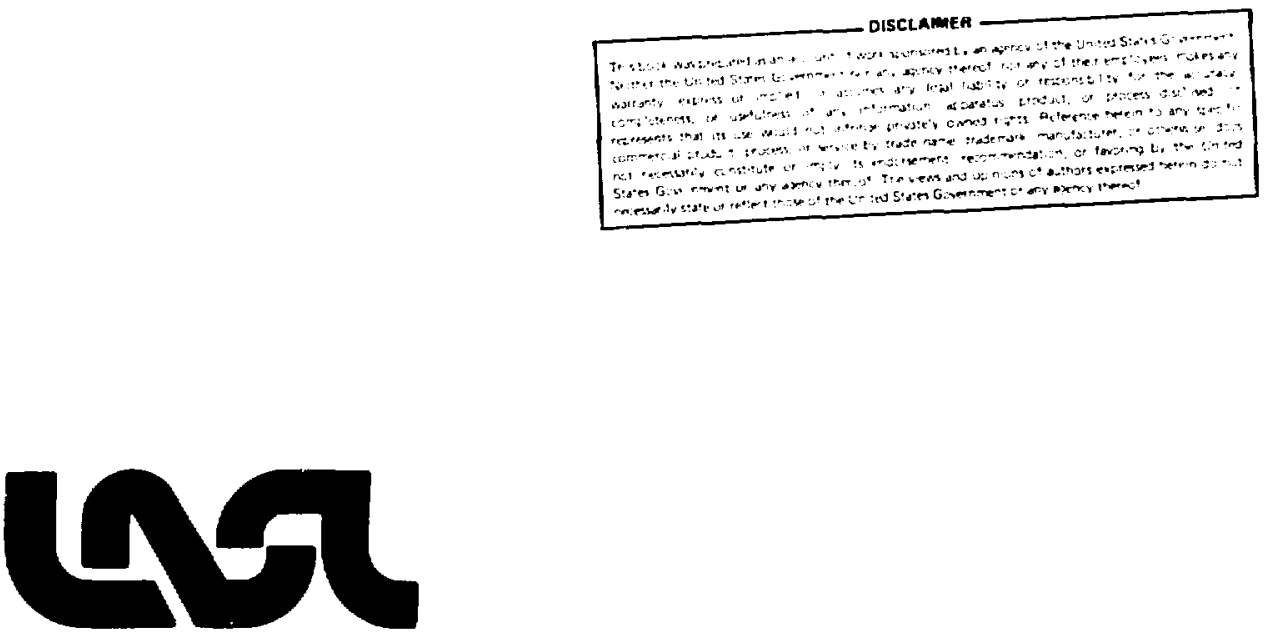
MATCHING BUNCHED BEAMS TO ALTERNATING GRADIENT FOCUS ING SYSTEMS

by

W. P. Lysenko

\begin{abstract}
A numerical procedure for generating phase-space distributions matched to alternating gradient focusing systems has been tested. For a smooth-focusing system a matched distribution can be calculated. With a particle tracing simulation code such a distribution can be followed wile adiabatically deforming the focusing forces until an alternating gradient configuration is reached. The distribution renains matched; that is, the final distribution is periodic with the structure period. This method is useful because it can produce distributions matched to nonlinear forces. This is a feature that elliptical distributions, with ellipse parameters obtained from the courant-Snyder theory, do not have. External nonlinearities, including nonlinear couplings, were included in our examples but spawe charge was not. This procedure is expected to work with space charge but will require a three-dimensional space charge calculation in the simulation code.
\end{abstract}

\title{
I. INTRODUCT ION
}

One problem in linear accelerator beam dynamics is the determination of matched phase-space distributions. In the smooth approximation in which the focusing forces are explicitly time independent, a phase-space distribution is said to be matched if it is constant in time. These atched or equilibrium distributions are desirable in an accelerator because they wake efficient use of available phase space. Matched distributions are important also in numerical simulation studies because their time evolution is known (they are constant). Emittance growth observed in a particle tracing simulation in wich a 
matched distribution is used as input would be purely numerical. Equilibrium distributions can be formed by taking functions of the single-particle Hamiltonian (which can include space charge)

$$
f(\vec{x}, \vec{p})=F(H(\vec{x}, \vec{p}))
$$

This procedure yields equilibrium distributions because the Hamiltonian is a conserved quantity for time-independent systems.

For periodic focusing systens such as alternating gradient fccusing systems, a matched phase-space distribution is one that is periodic in time with the same period as the focusing forces. Because the forces are time dependent, Eq. (1) cannot be used and another method of generating matched distributions must be used. In the absence of any nonlinearities, matched distributions can be determined for periodic syotems by the courant-snyder theory, which results in elliptical phase-space distributions. In this paper we present the results of some tests on a method that produces distributions that are matched to periodic focusing systems that include nonlinear forces. The procedure for obtaining such atched distributions is the following. An azimuthally symetric distribution matched to a swooth-focusing systen containing the axial nonlinearity and $r$ coupling terns is prepared by the numerical code RZED79, which uses Eq. (1). This distribution, represented by a collection of macroparticles, is followed in the particle tracing simulation code HOT while the focusing forces are adiabatically deformed until an alternating gradient configuration is reached. The distribution remains matched; that is, it evolves from a time-independent distribution to one that is periodic with the structure period, provided the change in the accelerator parameters is effected slowly enough. An alternating gradient structure is three-dimensional and because the present HOT code does not have three-dinengional apace-charge capabilities, the described calculations were done without space charge. The essential new feature studied is the tine dependence of the forces. Spacecharge forces or other features of the focusing forces could be included in the particle tracing code, and distributions matched to the new situation should result. 
II. DISTRIBUTIONS MATCHED TO SMOOTH-FOCUSING SYSTEMS

Distributions matched to a smooth-focusing system were generated using Eq. (1) with the following choice for the function $F$

$$
F(H) \sim \begin{cases}H_{0}-H, & H_{0}-H \geqslant 0 \\ 0 & , \\ H_{0}-H<0\end{cases}
$$

in which $H_{0}$ is a constant. This is one of the functions used in the one-degree-of-freedom work by Gluckstern, Chasman, and Crandall.1 In the present case we use a two-degree-of-freedom, r-z Hamiltonian to describe the nonrelativistic single-particle motion.

$H(\vec{x}, \vec{p})=\frac{1}{2 m} p^{2}+\frac{k}{2} r^{2}+\frac{k_{z}}{2} z^{2}-\frac{\pi k z \cot \phi_{s}}{3 \beta \lambda} z^{3}+\frac{\pi k z \cot \phi_{s}}{3 \beta \lambda} r^{2} z+e \phi(r, z)$

The coordinates $\vec{x}$ and momenta $\vec{p}$ are relative to the synchronous particle with $r^{2}=x^{2}+y^{2}$ and $p^{2}=p_{x}^{2}+p_{y}^{2}+p_{z}^{2}$. The synchronous phase is $\phi_{s}$ and the space-charge potential is $\phi(r, z)$. Except for the inclusion of the axial nonlinearity term $\left(z^{3}\right)$ and the $r f$ coupling term $\left(r^{2} z\right)$, the procedure for producing a matched distribution using the RZED code (the nonlinear version is called RZED79) has been previously described. ${ }^{2}$ with no space charge $(\phi=0)$, the procedure is straightforward.

We started with the beam described in Table $I$. This beam was matched to a $2 B \lambda$ drift-tube linear accelerator described in Table II, assuming smooth focusing with a phase advance of $90^{\circ}$ per focusing period in all directions. We chose a large beam emittance to more clearly see nonlinear effects. (The normalized emittance in the $x$-direction is defined as the area in the $x-p_{x}$ plane divided by $\pi m c$. In teras of the angle variable $x^{\prime}=d x / d(\rho, t)$, the normalized emittance is the area in the $x-x^{\prime}$ plane multiplied by $\left.\beta y / \pi_{\bullet}\right)$ The beam radius is approximately $\beta \lambda / 7, a$ value that is larger than would be used in ary real accelerator. (The transverse normalized acceptance is $\gamma r^{2} /(n \lambda)$ where $r$ is the bore radius, $\sigma$ is the phase advance per focusing period, $n$ is the number of $r f$ periods in a focusing period, and $\lambda$ is the $r f$ wavelength.) To enhance nonlinear effects even more, the initial bean was traced for 20 focusing periods wile the focusing strengths of the smooth-focusing forces were increased so that the final phase advances were $108^{\circ}$ in all three 
TABLE I

INITIAL BEAM PROPERTIES

INITIAL BEAM PROPERTIES FOR 98\% OF BEAM

\begin{tabular}{lcc} 
& $90^{\circ}$ phase advance & $108^{\circ}$ phase 8 dvance \\
\cline { 2 - 3 }$r$ & $2.2 \mathrm{~mm}$ & $2.0 \mathrm{~mm}$ \\
$r / \beta \lambda$ & 0.14 & 0.13 \\
$z_{\min }$ & $-2.2 \mathrm{~mm}$ & $-2.0 \mathrm{~mm}$ \\
$z_{\max }$ & $1.7 \mathrm{~mm}$ & $1.6 \mathrm{~mm}$ \\
$\Delta \phi_{\min }$ & $-38.8^{\circ}$ & $-36.3^{\circ}$ \\
$\Delta \phi_{\max }$ & $50.1^{\circ}$ & $44.8^{\circ}$ \\
$x$ & $2.7 \mathrm{~mm} \cdot \operatorname{mrad}$ & $2.6 \mathrm{~mm} \cdot \operatorname{mrad}$
\end{tabular}

TABLE II

ACCELERATOR PARAMETERS

Injection energy

'requency

Transverse phase advance

Longitudinal phase advance

Synchronous phase
$250 \mathrm{keV}$

$440 \mathrm{MHz}$

$90^{\circ}-108^{\circ}$

$90^{\circ}-108^{\circ}$

$-46.4^{\circ}$

directions. The resulting beam is described in the second column of Table $I$. This new "initial" beam was then used as che starting point in the experinent to generate the time-dependent matched beam. The $108^{\circ}$ tune is close to the nonlinear $2 \sigma_{x}+\sigma_{z}=360^{\circ}$ resonance so that matching to the nonlinearity becomes more important.

\section{PARAMETER VARIATION IN PARTICLE TRACIMG}

If the coordinates of a particle, measured relative to the synchronous particle, are $x, y$, and $z$ then the external forces on the particle in a periodic focusing system are functions of $x, y, s(t)+x$, and $t$ where $s(t)$ is the position of the synchronous particle. These functions are periodic in the last two variables (in our exanple the periods are $4 \beta \lambda$ and $\tau$ where $\tau$ is the rf period). We used a simplified wodel in which the forces are functions of $x, y, z$, and $t$, periodic in the time variable. This approximation neglects 
initial
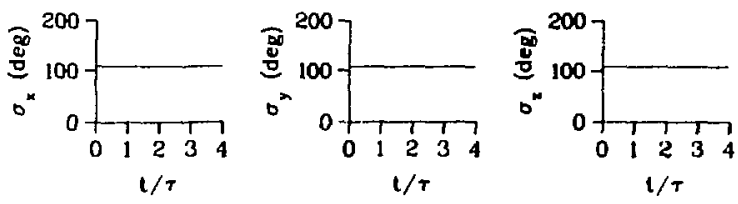

intermediate
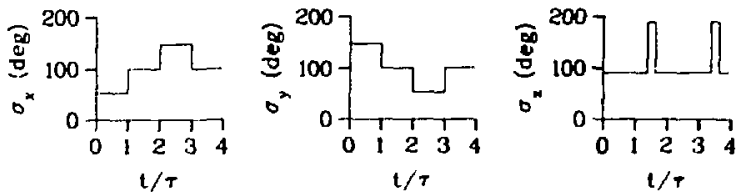

final
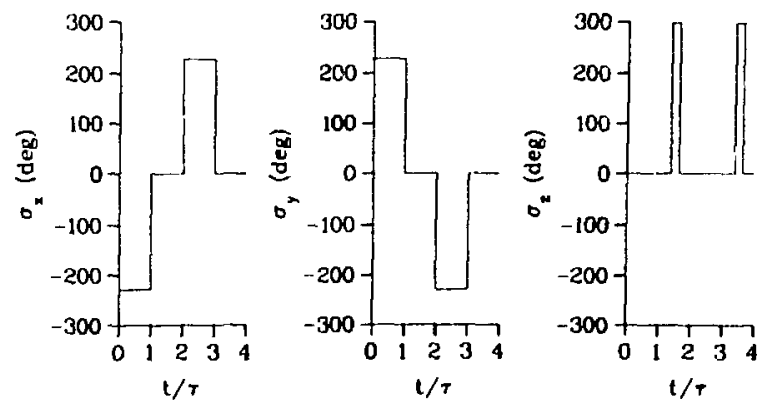

Fig. 1. The focusing strengths in the three directions are shown as function: of time over one focusing period for the initial, an intermediate, and the final configuration. The phase advance per period is always $108^{\circ}$ in all directions. the fact that different par-

ticles arrive at a given point at different times and also neglects the fact that the forces in the gap are time dependent. Had we included space-charge forces, the above properties would apply to them also, provided the distribution were matched. Figure 1 shows the strengths of the linear parts of the focusing forces in the three directions over one focusing period (four rf cycles). The focusing sirengths are expressed in terms of instantaneous phase advances per focusing period. For example, in the $x$-direction, an instantaneous phase advance $\sigma_{x}(t)$ at time $t$ means that if the focusing strength were held constant over one focusing period

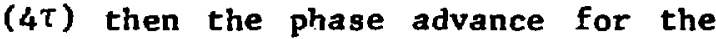
period would be $\sigma_{x}(t)$. A negative value for the instantaneous phase advance means the force defocuses and would produce a phase advance of $-\sigma_{x}(t)$ if its direction were reversed. The focusing period in Fig. 1 starts with an $x$-defocusing lens, followed by a drift, followed by an $x$-focusing lens, followed by a second drift. The lens-filling factor is one-half so that the lens lengths are $\beta \lambda$. At the centers of the drifts are rf gaps of length $B \lambda / 4$. 
The alternating gradient configuration is achieved as follows. In the first focusing period of the simulation, the focusing strengths are constant over the period and correspond to a phase advance of $108^{\circ}$ in all three directions. In subsequent focusing periods, the focusing strength in the $x$-direction is decreased in the region of the $x$-defocusing lens and increased in the region of the $x$-focusing lens so that the instantaneous phase adavances $\sigma_{x}(t)$ in these regions are some new values $\sigma_{1}$ and $\sigma_{2}$, respectively. In the drifts between the lenses, the focusing strength is set so that the instantaneous phase advance there is $\left(\sigma_{1}+\sigma_{2}\right) / 2$. In the $y$-direction, the focusing strengths are set so that the instantaneous phase advances are ${ }_{2}$, $\sigma_{1}$, and $\left(\sigma_{1}+\sigma_{2}\right) / 2$ in the three regions, respectively. In the $z$-direction, the instantaneous phase advance in the gap region is increased to a value $\sigma_{5}$ and the instantaneous phase advance in the region between the gaps is decreased to a value $\sigma_{4^{\circ}}$ At every focusing period, the strengths are changed by a small amount until finally $\sigma_{1}$ and $\sigma_{2}$ are equal in magnitude but opposite in sign and the drifts contain no transverse forces. In the axial direction, the final configuration has forces confined to the gap region.

Figure 2 shows curves of constant phase advance in the $\sigma_{1}-\sigma_{2}$ plane. The line $\sigma_{2}=\sigma_{1}$ corresponds to smooth focusing and the line $\sigma_{2}=-\sigma_{1}$ corresponds to sternating gradient focusing. Figure 3 shows curves of

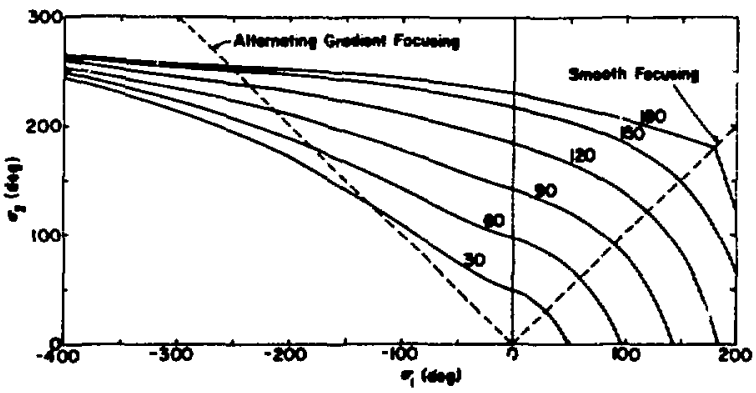

Fig. 2. Curves of constant transverse phase advance (labeled by phase advance in degrees per focusing period). The instantaneous phase sdvances in the regions of the defocusing and the focusing lenses are $\sigma_{1}$ and $\sigma_{2}$, respectively.

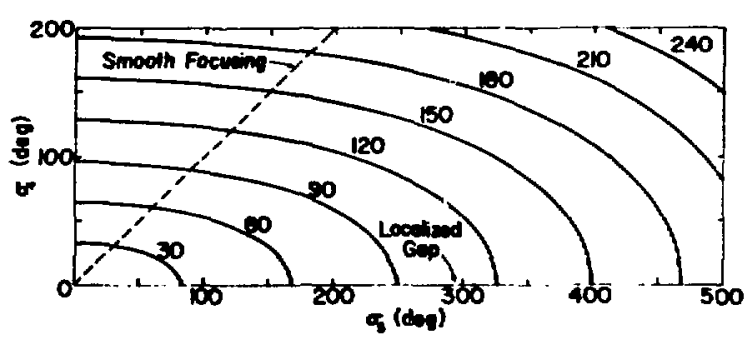

Fig. 3. Curves of constant longitudinal phase advance (labeled by whase advance in degrees per focusing period). The instantaneous phase advances in the rf gap and in the intergap region are $\sigma_{5}$ and $\sigma_{4}$, respectively. 

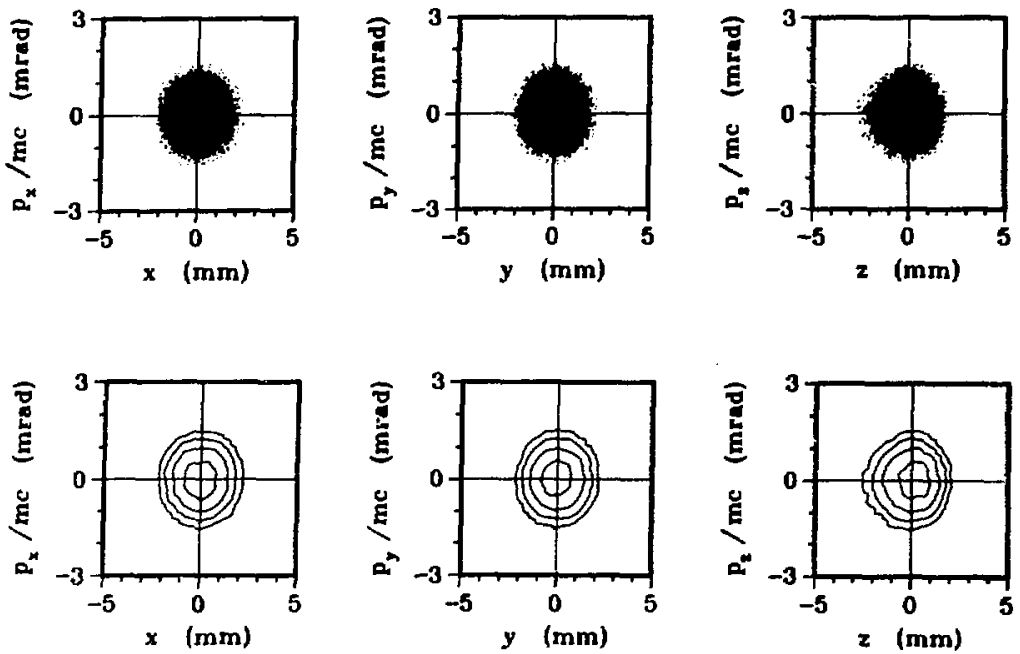

Fig. 4. Matched beam at focusing period number 20. The structure at this point is smooth focusing with phase advances per period of $108^{\circ}$ in al1 directions. The scatter plots show the projections of the sixdimensional phase-space coordinates of the 10000 macroparticles onto the two-dimensional phase-space planes. The contour plots show contours of equal density of points in the two-dimensional projections. The contours show densities of $0.7,0.3,0.1$, and 0.02 in units of the maximum density.

constant phase advance in the $\sigma_{5}-\sigma_{4}$ plane. The line $\sigma_{4}=\sigma_{5}$ corresponds to smooth focusing and the line $\sigma_{4}=0$ corresponds to the axial forces localized to the rf gap region. In the example in this paper, we varied the parameters $\sigma_{1}, \sigma_{2}, \sigma_{4}$, and $\sigma_{5}$ so that the phase advances were a1ways $108^{\circ}$ in a11 directions.

IV. PARTICLE TRACING RESULTS

The initial matched phase-space distribution was represented in the particle tracing code HOT by 10000 macroparticles. Figure $4 \mathrm{a}$ shows the three two-dimensional phase-space projections at focusing period 20 , where the focusing forces are still smooth and correspond to a phase advance of $108^{\circ}$. Figure $4 \mathrm{~b}$ shows the contours of equal density in the same phase planes. In this and all contour plots of this paper, the contours represent densities of $0.7,0.3,0.1$, and 0.02 in units of the maximum phase-space density. The axial phase-space shape is not elliptical because the beam is matched to the very nonlinear axial forces. This distribution was traced from this point with acceleration still turned off while bringing the focusing forces to the 

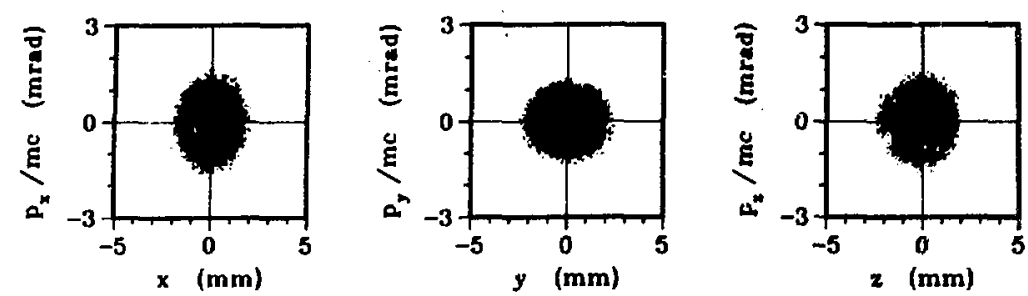
(a) at center of $x$-defocusing lens
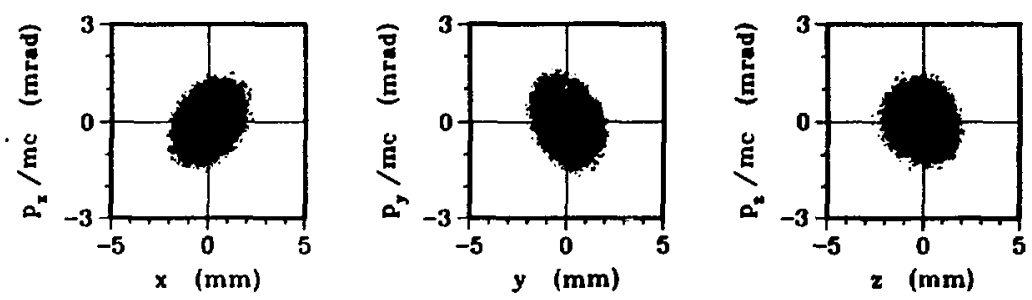

(b) at exit of first
rf gap
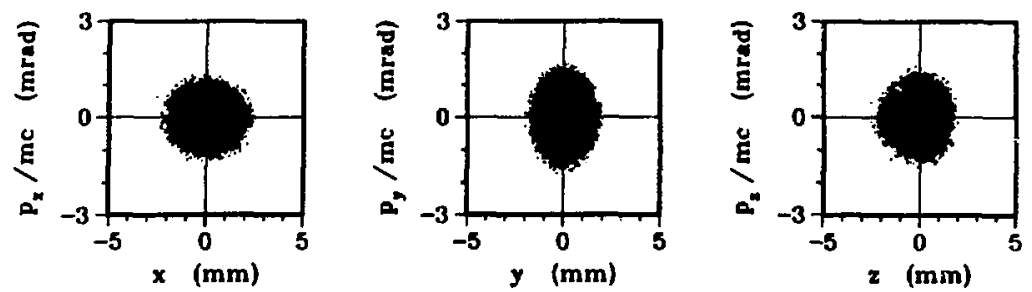

(c) at center of $\mathrm{x}$-focusing lens
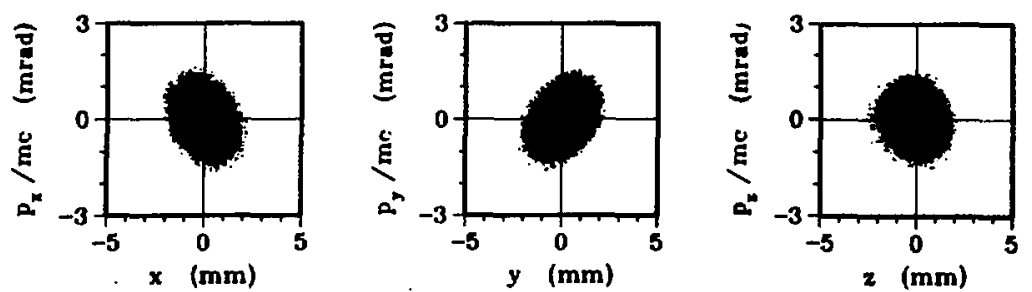

(d) at exit of
second rf gap

Fig. 5. Scatter plots for matched bean at focusing period number 37 . The accelerator structure at this time corresponds to the "internediate" configuration of Fig. 1 . 

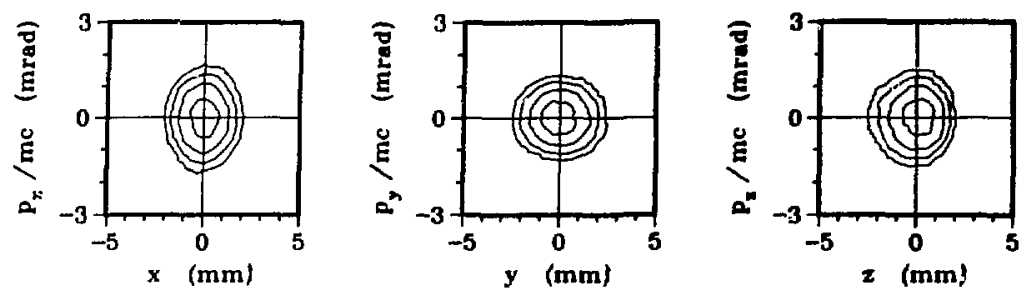
(a) at center of
- $x$-defocusing lens
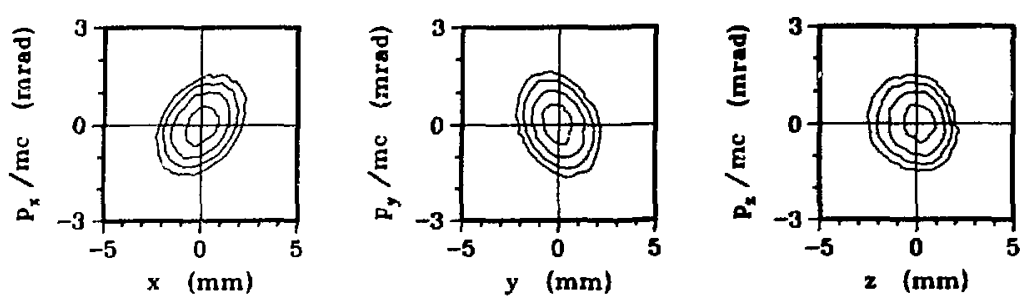
(t) at exit of first rf gap
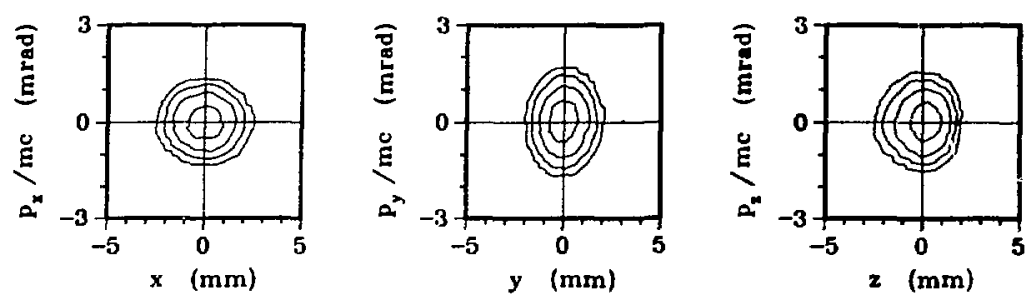

\section{(c) at center of $x$-focusing lens}
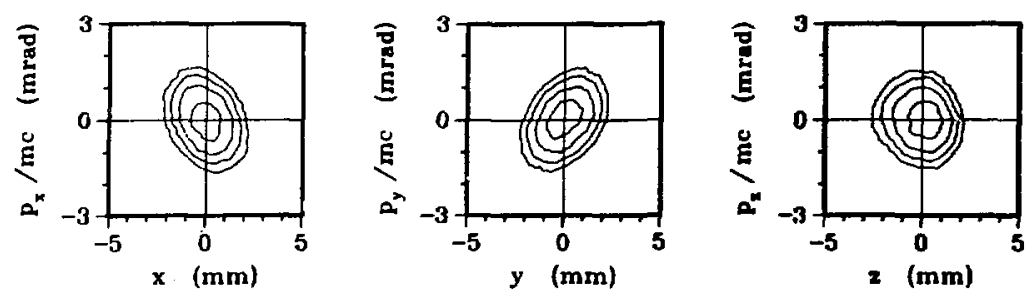

(d)

at exit of second rf gap

Fig. 6. Contour plots for matched beam at focusing period number 37. 

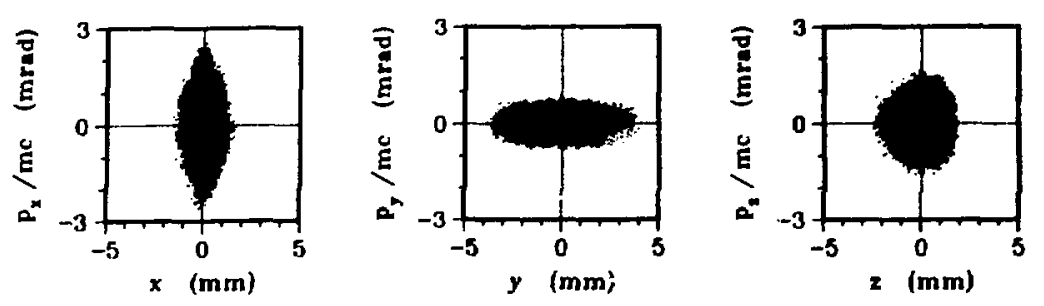
(a) at center of $x$-defocusing lens
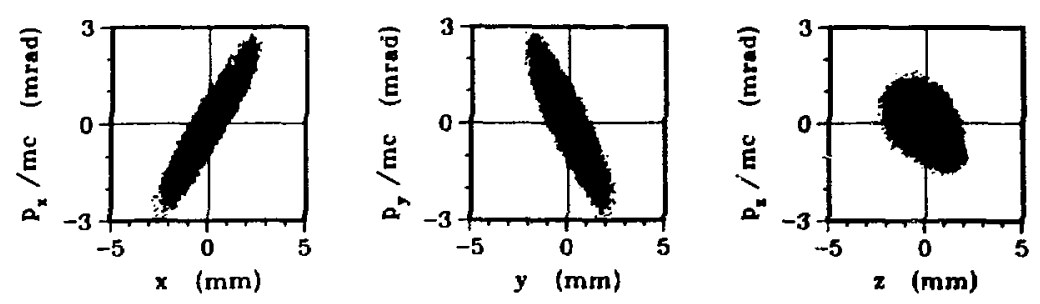
(b) at exit of first $r f$ gap
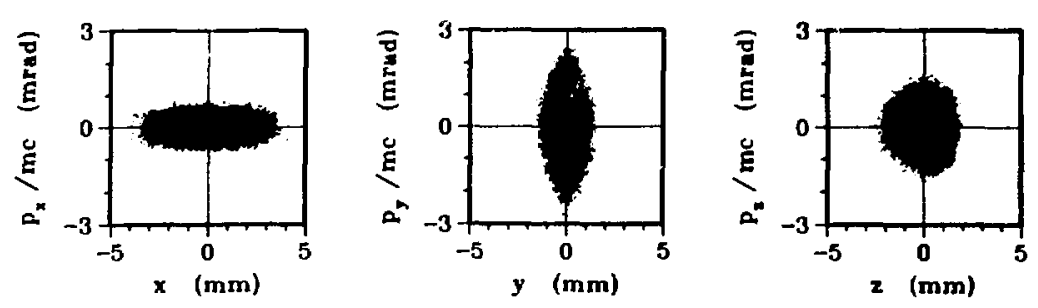
(c) at center of $x$-focusing lens
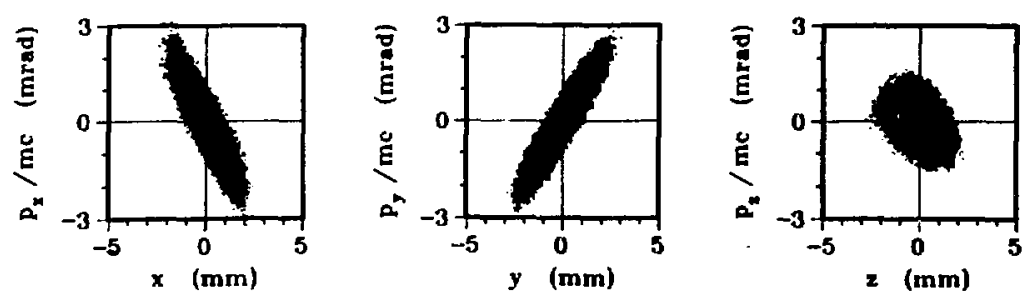
(d) at exit of second rf gap

Fig. 7. Scatter plots for matched bean at focusing period number 121. The accelerator structure at this time corresponds to alternating gradient focusing as shown in the "final" configuration of Fig. 1. 

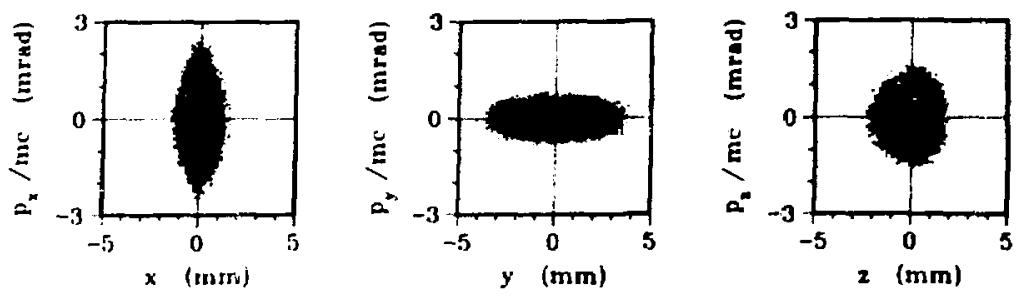

(a) at center of
$x$-defocusing lens
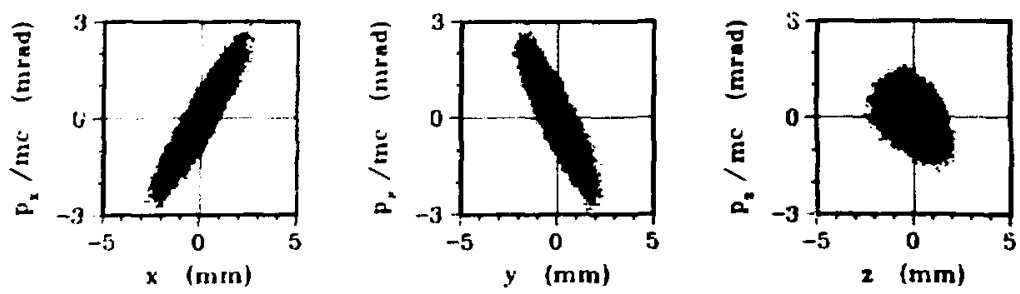

(b) at exit of first If gap
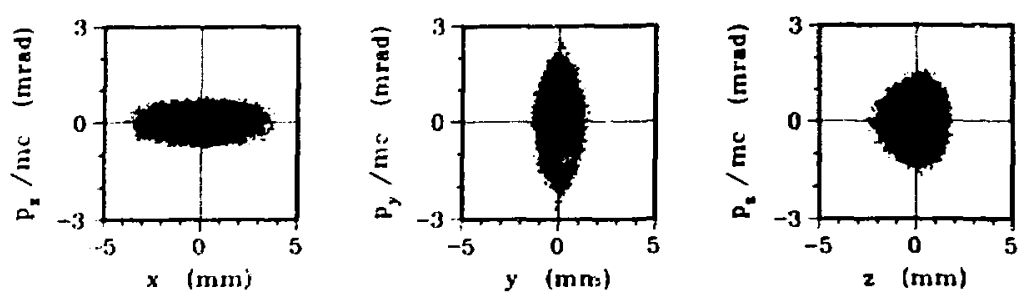

(c) at center of $x$-focusing lens
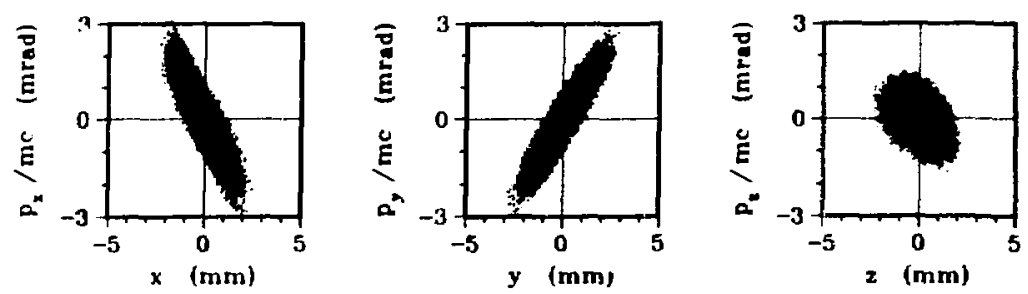
(d) at exit of second if gap

Fig. 8. Scatter plots for matched beam at focusing period number 126 . The accelerator structure has been held fixed in the alternating gradient configuration since focusing period number $12 i$. Note that corresponding phase-space projections are similar to those in Fig. 7 . 

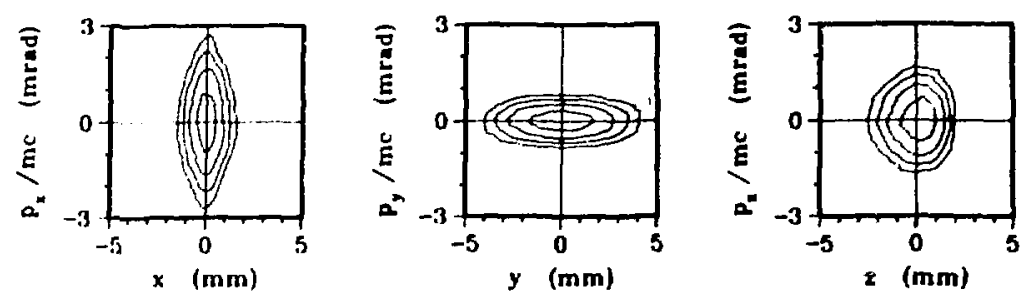
(a) at center of $x$-defocusing lens
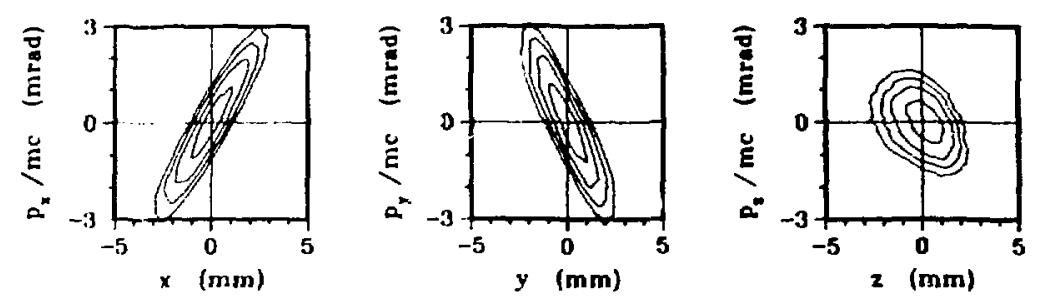
(b) at exit of first rf gap
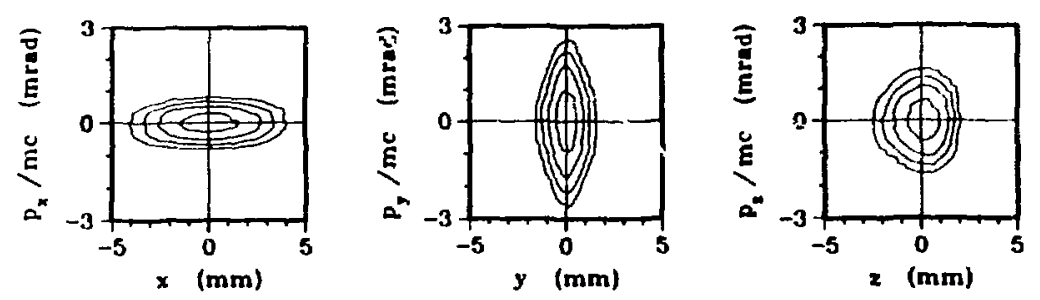
(c) at center of $x$-focusing lens
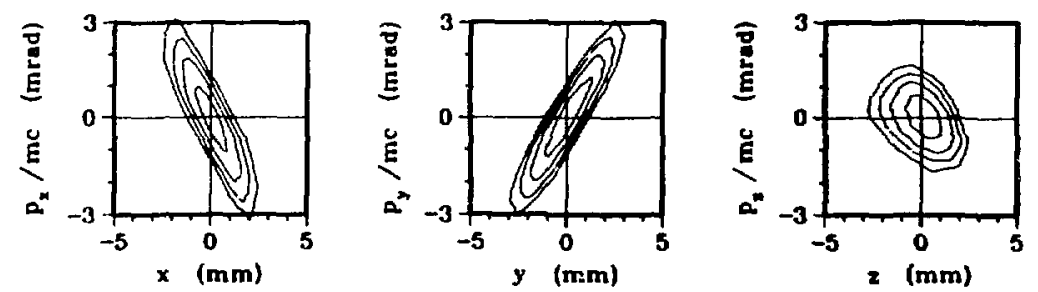

(d) at exit of
second rf gap

Fig. 9. Contour plots for matched beam at focusing period number 121 . 

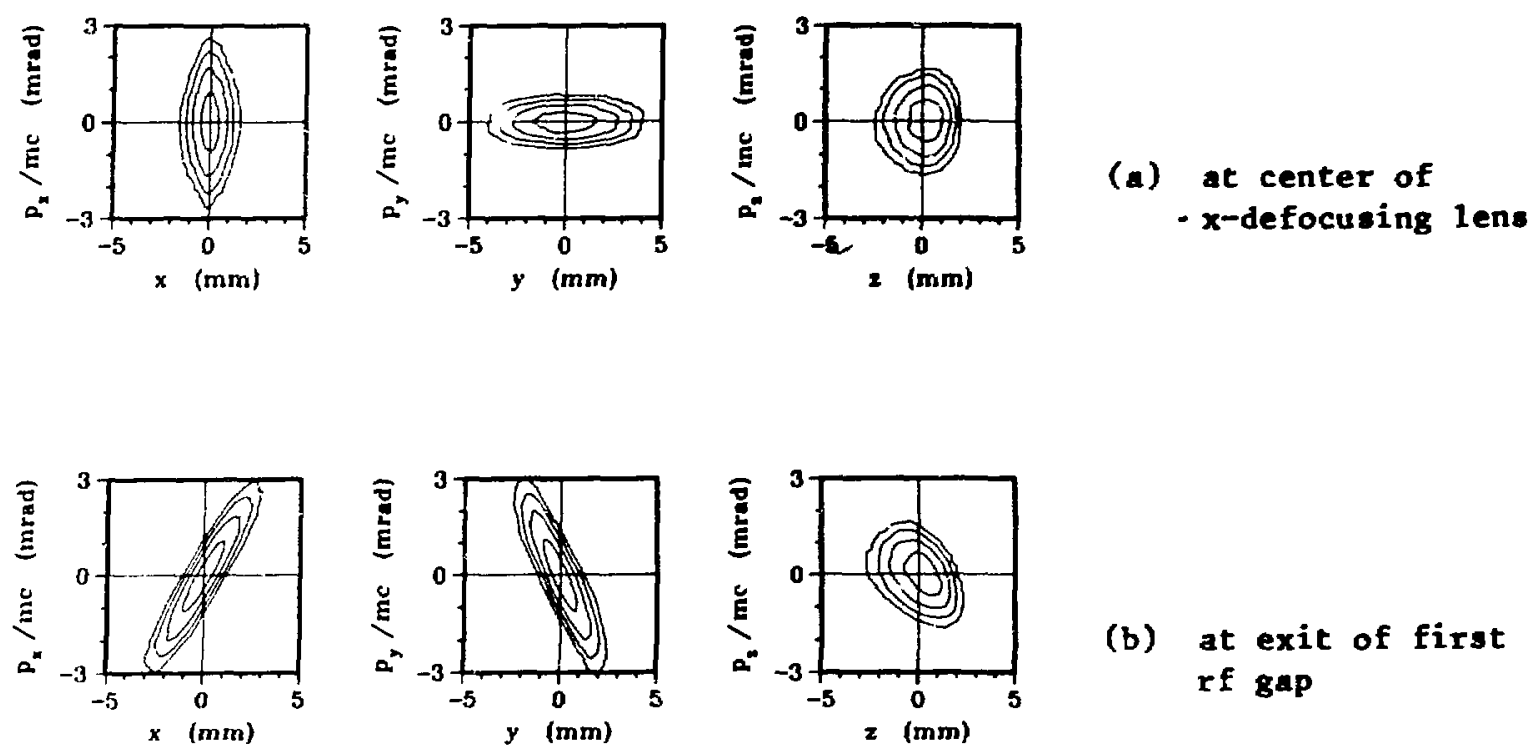
(b) at exit of first rf gap
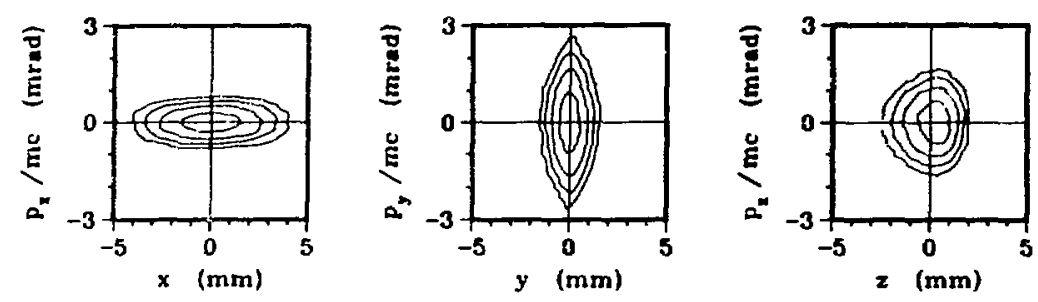

(c) at center of $x$-focusing lens
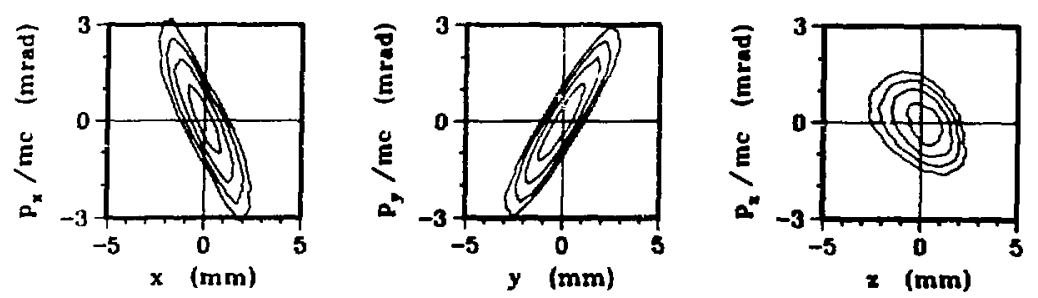
(d) at exit of second $r f$ gap

Fig. 10. Contour plots for matched beam at focusing period nubber 126. Note that corresponding phase-space projections are sinilar to those in Fig. 9 . 
final alternating gradient configuration in 100 focusing periods. This was done maintaining the phase advance per period at $108^{\circ}$ in all directions. Figures 5 and 6 show the scatter and contour plots for period 37, which corresponds to the "intermediate" configuration of Fig. 1. These plots show the three phase-space projections at the center of the $x$-defocusing lens, at the exit of the following $r f$ gap, at the center of the $x$-focusing lens, and at the exit of the second $r f$ gap of the focusing period.

The variation in the distribution over the focusing period is already evident. At period number 120 , the final alternating gradient configuration is reached and the accelerator parameters are held fixed as the distribution is followed for 20 more focusing periods. Figures 7 and 9 are the scatter and contour plots at focusing period 121. In this final configuration, the distribution undergoes large changes over a focusing period. The transverse beam size ratio between the focusing and defocusing lenses is 2.8. The maximum trangverse beam dimengion (at the center of the focusing lens) is 1.9 times the diameter of the smocth-focused beam of the same phase advance. Figures 8 and 10 show the scatter and contour plots five focusing periuds later, at period 126. The distributions, at times an integral number of focusing periods apart, are very similar. This is the desired result; that is, the distribution is periodic.

Figure 11 shows the normalized emittance in the $x$-direction as a function of the focusing-period number. The emittance values are those that contain $98 \%$ of the beam. The emittances are plotted at the centers of the lenses and at the exits of the rf gaps. Because the phase-space projections are not elliptical, the usual ellipse-fitting, area-measurenent procecure is not adequate. We used a bin-counting procedure, which was less shape dependent. The phase-space region containing the particles was divided into rectangular bins by a uniform rectangular mesh that enclosed the particle distribution. The size and orientation of this mesh was chosen by ellipse fitting. We discarded bins containing one particle and deterained the number of particles in the remaining bins. Then we discarded bins containing two particles and again determined the number of particles rearining. This procedure was continued with higher bin-population values until the desired number of particles (98\% of total) remained. The enittance value was the total area of the remaining bins divided by $\pi$. The statistical error in the 98\% bin-counting enittance of the initial bean when the distribution is 


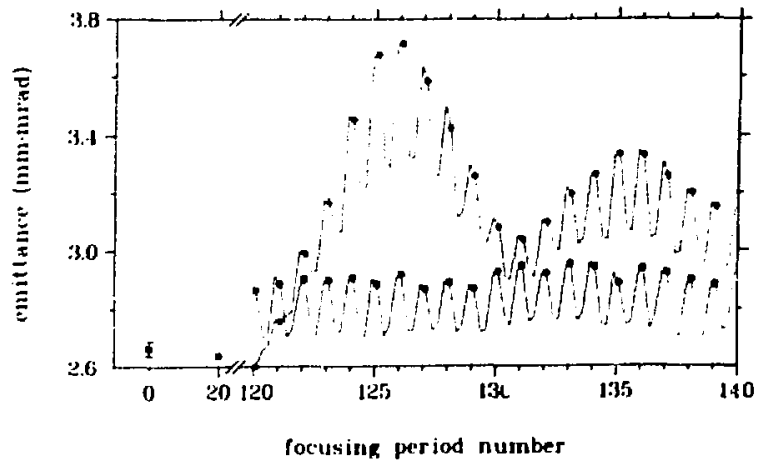

Fig. 11. The normalized emittance in the $x$-direction for $98 \%$ of the beam as a function of the focusing period number.

The emittances are evaluated at the centers of the lenses and at the exits of the $r f$ gaps with the curve markers indicating the emittance at the center of the $x$-defocusing lens. Square curve markers are used for the matched beam (lower curve) and circular curve markers are used for the uniform-in-six-

dimensiona 1-phase-space beam (upper curve). represented by 10000 macroparticles is 1.37 (error bar is indicated on the initial point in Fig. 11). This is the standard deviation in the emittance of a sample of ten $10000-$ particle representations of the desired distribution.

There are fluctuations in the $98 \%$ emittance of $\pm 3.5 \%$. The emittance is large in the defocusing lens and it is small in the focusing lens. Emittance values, at times an integral number of periods apart, are more nearly constant. The worst point in the twenty periods studied was at the $x$-defocusing lens in period 134 for which the emittance was 3.07 higher than at the corresponding point of period 121 . Because the emittance i approximately periodic, the emittance averaged over a period is a nearly constant quantity. The 98\%

emittance averaged in this way varies at most by +2.37 , at period 134, from the average at period 12l. Another kind of average that is even more constant is the $98 \%$ emittance averaged over the three directions $x, y$, and $z$. Averaged this way, the fluctuations over a period almost disappear. All 80 values of $\left(n_{x}+n_{y}+n_{z}\right) / 3$ over the 20 periods of the alternating gradient configuration lie in the interval $2.80 \mathrm{~mm} \cdot \mathrm{mrad} \pm 0.9 \%$. This is an experinental result that could not have been predicted because, for more than one degree of freedom, there is no known conservation law involving the enittances.

Figure 12 shows the the rms emittance in the $x$-direction as a function of the focusing-period number. The general behavior is similar to that of the 98\% emittance. The fluctuations are smaller $( \pm 2.4 \%)$ and the maximu change in the emittance averaged over a period is saller $(+1.27)$. For a linear 


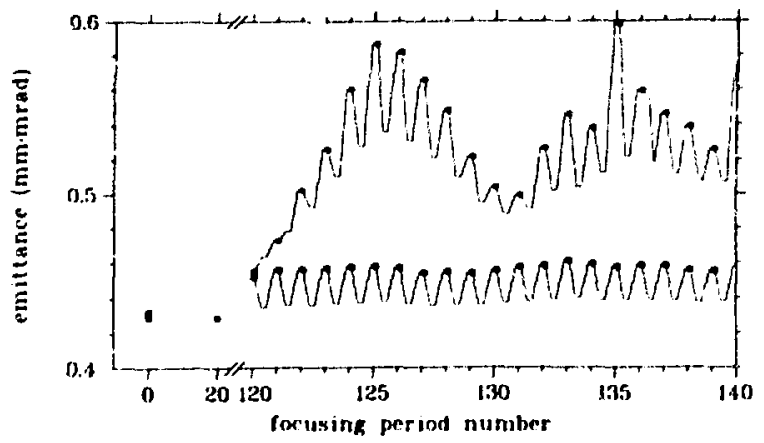

Fig. 12. The rms emittance in the $x$-direction as a function of the focusing period number. The emittances are evaluated at the centers of the lenses and at the exits of the rf gaps with the curve markers indicating the emittance at the center of the $x$-defocusing lens. Square curve markers are used for the matched beam (lower curve) and circular curve markers are uged for the uniform-in-sixdimensional-phase-space beam (upper curve). uncoupled system, the rus enittance is conserved for any distribution, even a mismatched distribution. The fluctuations observed are therefore caused by the nonlinearities we have introduced in the focusing forces.

For comparison, a uniformly filled ellipsoid in six-dimensional phase space was prepared with the Courant-Snyder parameters chosen to match to the linear part of the focusing forces. The emittances for this uniform beam were chosen so that the rms emittances for this uniform and the properly matched distribution described previously were the same. Figure 13 shows the phase space scatter and contour plots for the uniform beam. The beam is matched to the center of the
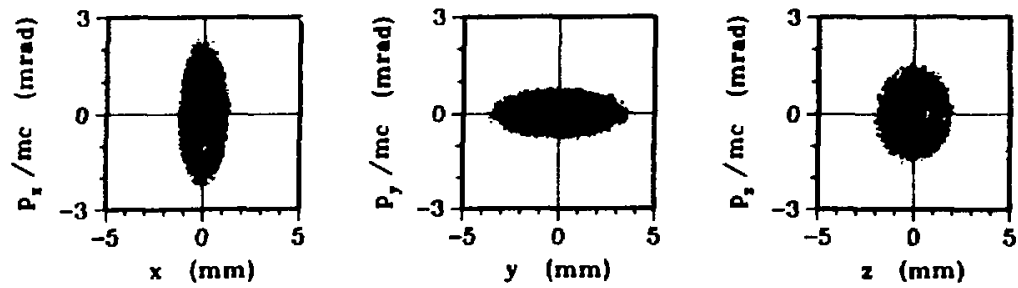

(a)
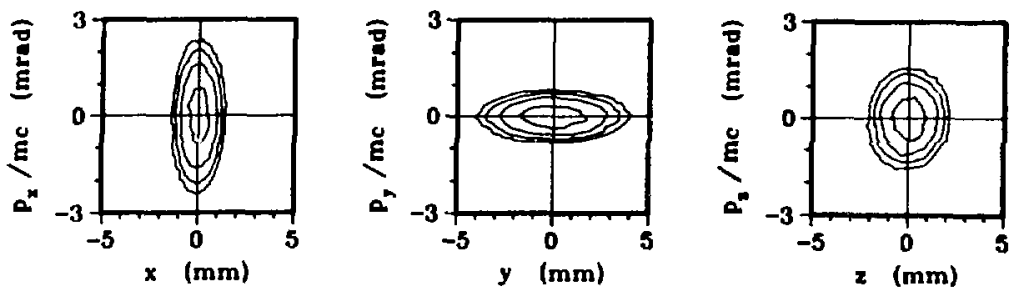

(b)

Fig. 13. Scatter and contour plots for a bean that is uniform in six-dimensional phase space. This beam is matched to the linear part of the focusing forces at the center of an $x$-defocusing lens. 
$x$-defocusing lens. The $98 \%$ transverse emittance of the uniform beam is 0.91 of that of the properly matched beam so the uniform beam sees slightly less nonlinear forces. Nevertheless, because the nonlinearities were not taken into account in preparing the uniform distribution, there is a mismatch. Figures 14 and 15 show the scatter and contour plots of the initially uniform distribution after five focusing periods. The $98 \%$ and rms emittance in the $x$-direction as a function of the focusing period number are shown in Figs. 11 and 12 along with the results for the matched beam. The mismatch leads to emittance growth.

The time evolution of the uniform beam can be understood to some degree because the tune is near the $2 \sigma_{x}+\sigma_{z}=360^{\circ}$ resonance. This resonance is excited by the $x^{2} z \cos (2 \pi t / \tau)$ term in the Hamiltonian. This term is the first harmonic of the rf coupling potential. Because $r f$ coupling is included in our model and because the beam is large, we expect the resonance effect to be large. The tune $\left(\sigma_{x}=\sigma_{z}=108^{\circ}\right)$ is such that the resonant combination of phase advances is $36^{\circ}$; that is, $2 \sigma_{x}+\sigma_{z}-360^{\circ}=36^{\circ}$. According to first-order perturbation theory, the amplitudes of the oscillation in the $x$ - and $z$-directions should oscillate with a period of 10 focusing periods $\left(360^{\circ} / 36^{\circ}\right)$. Because of the spread of z-phases, the $x$-amplitude should increase for some particles and decrease for other particles. The $z$-amplitudes should behave similarly. Consequently, we expect the emittance in both the $x$ - and $z$-directions to increase. In the simulation, the $98 \%$ emittance in the $x$-direction increases by $31 \%$ in six focusing periods. After 10 focusing periods we expect the distribution to return to its or eginal form because it was matched to the linear forces. Because the perturbation theory result is not exact, the $x$ - and z-emittances in the simulation both decrease and reach a local minimum after 11 focusing periods, but do not return ta their original values. There is some average emittance growth. Bagining with period number 133, some particles of the uniform bean becone "1ost". This means a transverse coordinate has exceeded five times the initial bean radius. These "lost" particles are not counted in the eaittance measurement so that the late-time 987 emittances, which are determined by the outermost particles, are misleading. The rms emittance evolution of the uniform beam shown in Fig. 12 better shows the average eaittance growth. Because the bean studied was so large, in an actual accelerator, wany particles in the uniform beam would actually be lost starting with the second or third focusing period. 

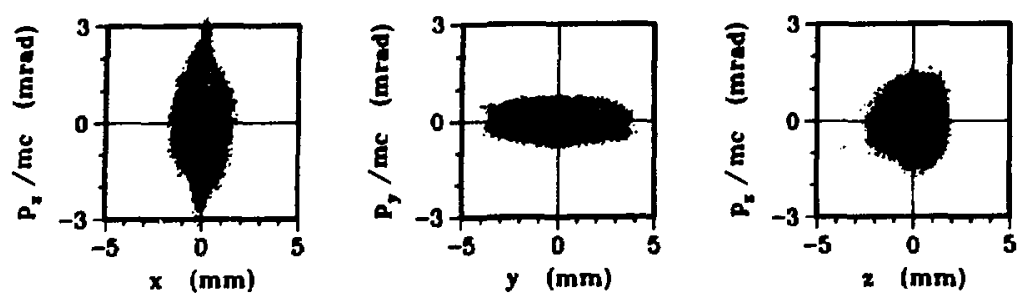
(a) at center of $x$-defocusing lens
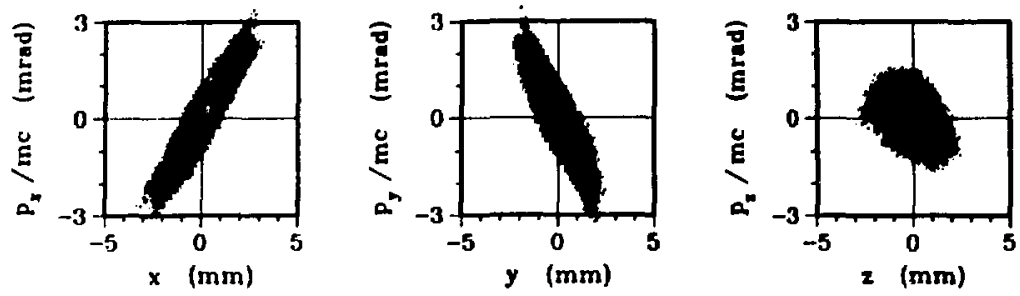

(b) at exit of first
rf gap
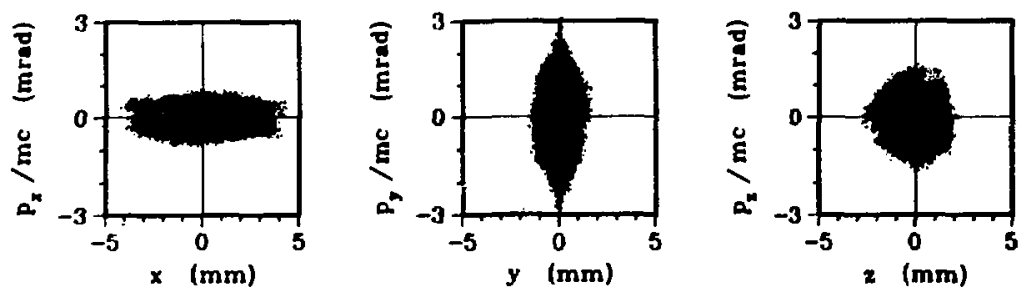
(c) at center of $x$-focusing lens
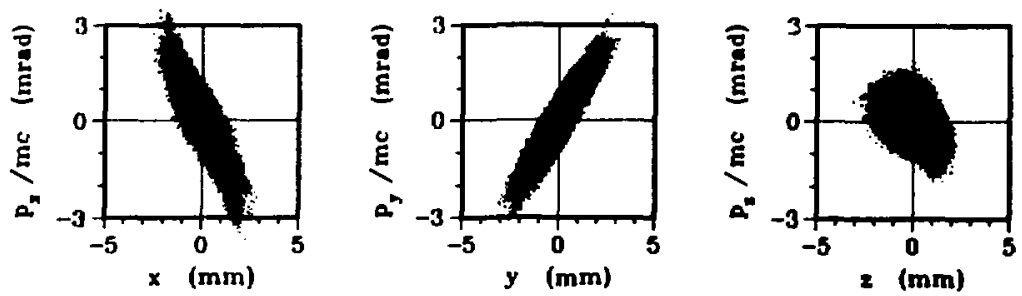
(d) at exit of second if gap

Fig. 14. Scatter plots for the initially uniform bean after five focusing periods. 

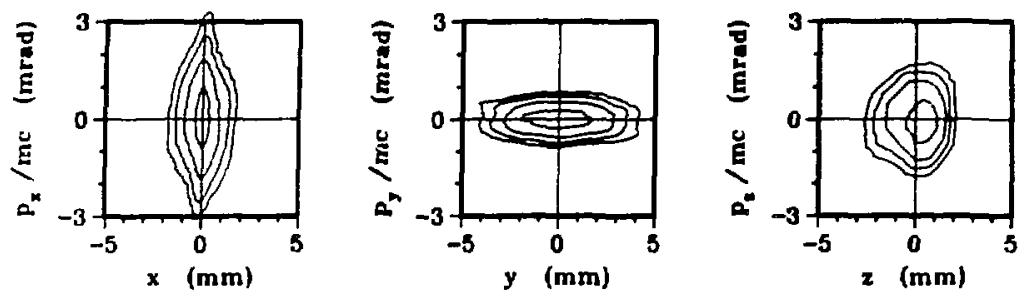
(a) at center of $x$-defocusing lens
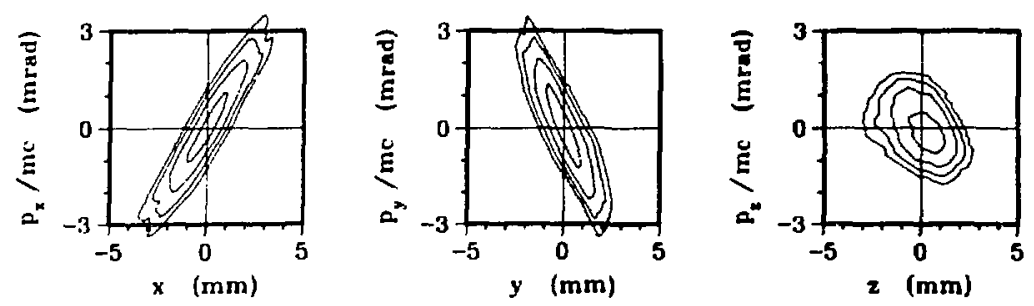
(b) at exit of first rf $\operatorname{gap}$
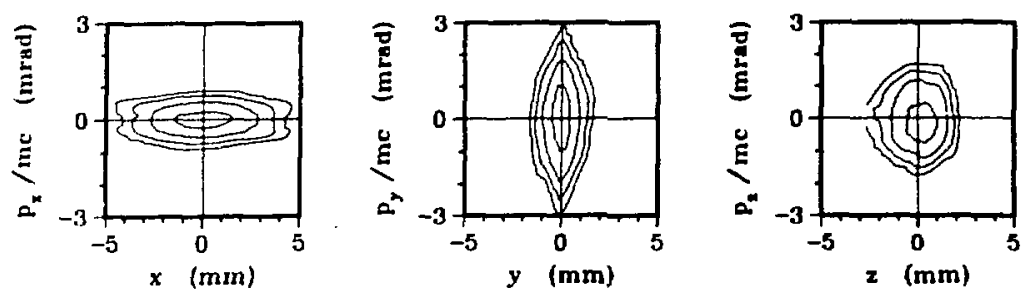

\section{(c) at center of $x$-focusing lens}
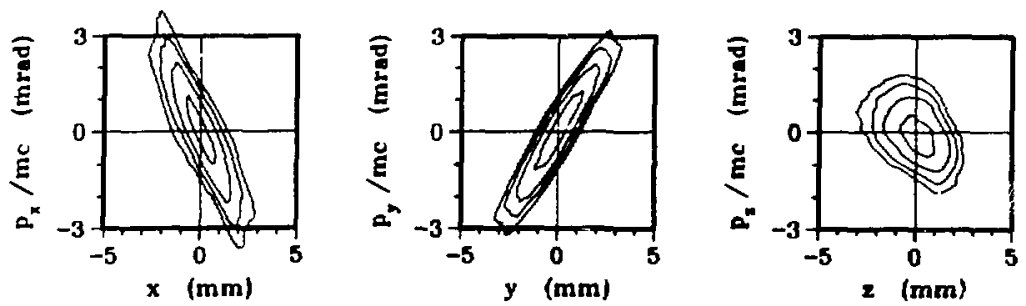
(d) at exit of second rf gap

Fig. 15. Contour plots for the initially uniform beat after five focusing periods. 
We have tested the following procedure. To produce a phase-space distribution that is matched to a given structure, we start with a structure for which an equilibrium distribution is known and deform the structure adiabatically into the desired structure, following the distribution with a particletracing code. Our example shows that we can obtain a distribution matched to an alternating gradient focusing structure, including the axial nonlinearity and nonlinear $r$ coupling forces, starting from a distribution matched to an azimuthally symetric, smcoth-focusing structure. This procedure resulted in a periodic distribution even though the beam was large and the tune was near a nonlinear resonance, which led to nonelliptical phase-space shapes. This matched distribution did not exhibit the emittance growth that occurred for an elliptical distribution with the Courant-Snyder parameters determined by the linear parts of the focusing forces. This result is encouraging because if it still works with space charge in the future three-dimensional particle tracing code, it means we will have distributions matched to a very realistic model of the accelerator.

This principle may also be applied to a physical apparatus to match injected beams into complex structures. We start with a structure for which it is easy to create: a matched distribution or for which the details of the phase-space distribution are not important and adiabatically deform into another structure. For example, if very strong focusing is used in the lowenergy section of an accelerator, the matched beam will be small. Therefore, the nonlinearities of the external focusing forces will be small and the space-charge effects will be sma11. (Although space-charge forces are large for a small beam, the relevant number is the ratio of space-charge forces to external focusing forces. This ratio is smail for the small beam that results from natching to strong focusing forces.) If the focusing forces are then gradually relaxed to a more reasonable level for the large remaining length of the accelerator, the beam bunch will grow and the distribution function will develop the necessary correlations between the various dimensions of the sixdimensional phase space that are now necessary for this large beam to match to the forces that contain nonlinearities and couplings arising from the external and space-charge forces. If this procedure works, we can reduce emittance growth (or any other change in the distribution function) that is not caused by random errors in the accelerator structure. 


\section{REFERENCES}

1. R. L. Gluckstern, R. Chasman, and K. Cranda11, "Stability of Phase-Space Distributions in Two-Dimensional Beams," Proc. 1970 Proton Linear Acielerator Conf., National Accelerator Laboratory, 1970, p. 823.

2. W. P. Lysenko, "Equilibrium Phase-Space Distributions and Space-Charge Limits in Linacs," Los Alamos Scientific Laboratory report LA-7010-MS (October 1977). 